\title{
Towards Understanding How the Lactone Moiety of Discodermolide Affects Activity
}

Simon J. Shaw*, Kurt F. Sundermann, Mark A. Burlingame, David C. Myles, B. Scott

Freeze, Ming Xian, Ignacio Brouard, and Amos B. Smith III*

\section{Supporting Information}

Full References

4 Mickel, S. J.; Sedelmeier, G. H.; Seeger-Weibel, M.; Berold, B.; Schaer, K.; Gamboni, R.; Chen, S.; Chen, W.; Jagoe, C. T.; Kinder, F. R. Jr; Loo, M.; Prasad, K.; Repic, O.; Shieh, W-C.; Wang, R-M.; Waykole, L.; Xu, D. D.; Xue, S. Org. Proc. Res. Dev. 2004, 8, 92-130.

5a Kinder, F. R., Jr.; Bair, K. W.; Chen, W.; Florence, G.; Francavilla, C.; Geng, P.; Gunasekera, S.; Lassota, P. T.; Longley, R. E.; Palermo, M. G.; Paterson, I.; Pomponi, S.; Ramsey, T. M.; Rogers, L.; Sabio, M.; Sereinig, N.; Sorensen, E.; Wan, R. Abstracts of Papers, 224th Meeting of the American Chemical Society, Boston, MA, Aug 18-22, 2002; American Chemical Society: Washington, DC, 2002; MEDI 236.

9b Burlingame, M. A.; Shaw, S. J.; Sundermann, K. F.; Zhang, D.; Petryka, J.; Mendoza, E.; Liu, F.; Myles, D. C.; LaMarche, M. J.; Hirose, T.; Freeze, B. S.; Smith, A. B. III Bioorg. Med. Chem. Lett. 2004, 14, 2335-2338.

9c Smith, A. B.; Freeze, B. S.; LaMarche, M. J.; Hirose, T.; Brouard, I.; Rucker, P. V.; Xian, M.; Sundermann, K. F.; Shaw, S. J.; Burlingame, M. A.; Horwitz, S. B.; Myles, D. C. Org. Lett. 2005, 7, 311-314.

9d Smith, A. B.; Freeze, B. S.; LaMarche, M. J.; Hirose, T.; Brouard, I.; Xian, M.; Sundermann, K. F.; Shaw, S. J.; Burlingame, M. A.; Horwitz, S. B.; Myles, D. C. Org. Lett. 2005, 7, 315-318.

Characterisation of final compounds : 


\section{Lactone 4}

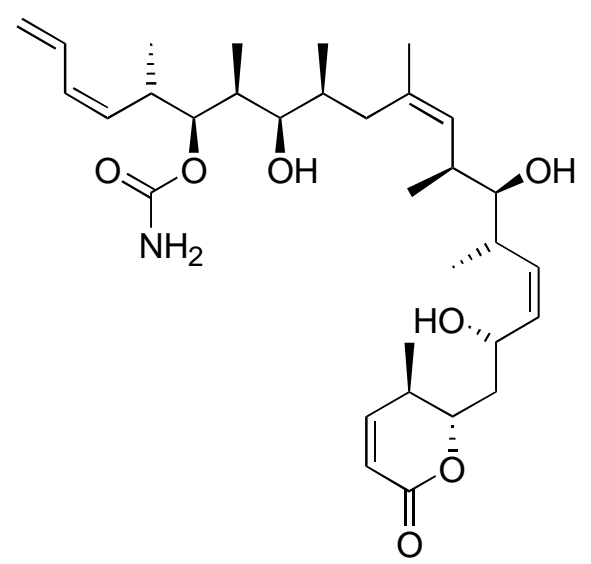

$[\alpha]_{\mathrm{D}}^{23}+44.1^{\circ}\left(c\right.$ 0.43); IR (film, $\left.\mathrm{CHCl}_{3}\right) 3418,3221,29672930,2872,1716,1599,1456$, 1393, 1324, 1256, $1041 \mathrm{~cm}^{-1} ;{ }^{1} \mathrm{H}$ NMR $\left(500 \mathrm{MHz}, \mathrm{CDCl}_{3}\right) \delta 6.75(\mathrm{dd}, J=9.8,2.5 \mathrm{~Hz}$, 1H), 6.67 (ddd, $J=16.8,11.0,11.0 \mathrm{~Hz}, 1 \mathrm{H}), 6.08$ (dd, $J=11.0,11.0 \mathrm{~Hz}, 1 \mathrm{H}), 5.87$ (dd, $J$ $=9.8,2.4 \mathrm{~Hz}, 1 \mathrm{H}), 5.56(\mathrm{dd}, J=10.5,10.1 \mathrm{~Hz}, 1 \mathrm{H}), 5.45-5.38(\mathrm{~m}, 2 \mathrm{H}), 5.25(\mathrm{dd}, J=$ 16.8, $1.9 \mathrm{~Hz}, 1 \mathrm{H}), 5.15$ (d, $J=10.2 \mathrm{~Hz}, 1 \mathrm{H}), 5.08$ (br s, 2H), 4.98 (d, J = $10.0 \mathrm{~Hz}, 1 \mathrm{H})$, $4.73(\mathrm{dd}, J=8.2,4.0 \mathrm{~Hz}, 1 \mathrm{H}), 4.50-4.44(\mathrm{~m}, 1 \mathrm{H}), 4.30(\mathrm{ddd}, J=10.2,10.2,2.1 \mathrm{~Hz}, 1 \mathrm{H})$, $3.15(\mathrm{ddd}, J=6.8,6.8,3.6 \mathrm{~Hz}, 1 \mathrm{H}) 3.10-3.05(\mathrm{~m}, 2 \mathrm{H}), 2.86(\mathrm{~d}, J=5.3 \mathrm{~Hz},) \mathrm{H}), 2.72(\mathrm{~d}, J$ $=5.4,2.6 \mathrm{~Hz}, \mathrm{OH}), 2.66(\mathrm{~d}, J=6.6 \mathrm{~Hz}, \mathrm{OH}), 2.63(\mathrm{br} \mathrm{ddd}, J=13.4,6.6,6.6,3.3 \mathrm{~Hz}$, $1 \mathrm{H}), 2.49-2.43(\mathrm{~m}, 1 \mathrm{H}), 2.32-2.21(\mathrm{~m}, 1 \mathrm{H}), 1.81-1.62(\mathrm{~m}, 6 \mathrm{H}), 1.61(\mathrm{~m}, 3 \mathrm{H}), 1.12(\mathrm{~d}, J=$ $7.2 \mathrm{~Hz}, 3 \mathrm{H}), 1.02$ (d, $J=7.0 \mathrm{~Hz}, 3 \mathrm{H}), 0.96$ (d, $J=6.8 \mathrm{~Hz}, 3 \mathrm{H}), 0.89$ (d, $J=6.6 \mathrm{~Hz}, 3 \mathrm{H})$, $0.78(\mathrm{~d}, J=6.8 \mathrm{~Hz}, 3 \mathrm{H}), 0.74(\mathrm{~d}, J=7.0 \mathrm{~Hz}, 3 \mathrm{H}) ;{ }^{13} \mathrm{C} \mathrm{NMR}\left(125 \mathrm{MHz}, \mathrm{CDCl}_{3}\right) \delta 163.6$, $157.3,152.2$, 133.1, 133.0, 132.9, 132.5, 132.1, 130.1, 129.5, 119.2, 117.5, 79.7, 78.8, 78.4, 74.9, 62.0, 40.9, 37.5, 36.0, 35.2, 35.0, 33.6, 33.4, 33.2, 22.2 , 18.7, 17.1, 16.5, 15.6, 14.5, 7.9; high resolution mass spectrum $\left(\mathrm{ES}^{+}\right) \mathrm{m} / \mathrm{z} 584.3571\left[(\mathrm{M}+\mathrm{Na})^{+}\right.$; calcd for $\mathrm{C}_{32} \mathrm{H}_{51} \mathrm{NNaO}_{7}$ : 584.3563].

\section{Lactone 5}




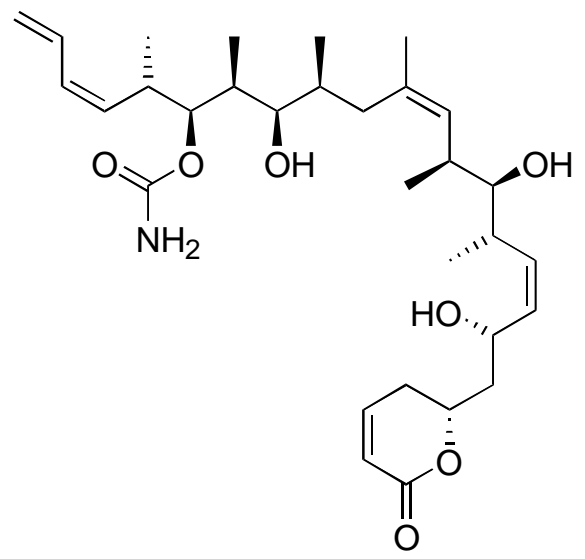

$[\alpha]_{\mathrm{D}}^{23}+53.4(\mathrm{c} 0.213, \mathrm{MeOH}) ;$ IR 3420, 2965, 1709, $1390 \mathrm{~cm}^{-1} ;{ }^{1} \mathrm{HNMR}(500 \mathrm{MHz}$, $\left.\mathrm{CD}_{3} \mathrm{OD}\right) \delta 7.05(\mathrm{~m}, 1 \mathrm{H}), 6.67(\mathrm{~m}, 1 \mathrm{H}), 6.08(\mathrm{dd}, J=11.0,11.0 \mathrm{~Hz}, 1 \mathrm{H}), 5.97(\mathrm{dd}, J=2.0$, $9.6 \mathrm{~Hz}, 1 \mathrm{H}), 5.67$ (dd, $J=10.5,10.5 \mathrm{~Hz}, 1 \mathrm{H}), 5.47$ (dd, $J=10.8,10.8 \mathrm{~Hz}, 1 \mathrm{H}), 5.40$ (dd, $J=8.6,10.8 \mathrm{~Hz}, 1 \mathrm{H}), 5.28$ (d, $J=16.8 \mathrm{~Hz}, 1 \mathrm{H}), 5.17(\mathrm{~d}, J=10.1 \mathrm{~Hz}, 1 \mathrm{H}), 4.94(\mathrm{~d}, J=$ $9.8 \mathrm{~Hz}, 1 \mathrm{H}), 4.86$ (br s, 2H), $4.84(\mathrm{~m}, 1 \mathrm{H}), 4.68(\mathrm{~m}, 1 \mathrm{H}), 4.53$ (dd, $J=8.6,8.6 \mathrm{~Hz}, 1 \mathrm{H})$, $3.21-3.14(\mathrm{~m}, 2 \mathrm{H}), 3.10(\mathrm{dd}, J=2.5,8.8 \mathrm{~Hz}, 1 \mathrm{H}), 2.73(\mathrm{~m}, 1 \mathrm{H}), 2.45-2.40(\mathrm{~m}, 2 \mathrm{H}), 2.28$ $(\mathrm{m}, 1 \mathrm{H}), 1.87-1.65(\mathrm{~m}, 6 \mathrm{H}), 1.66(\mathrm{~s}, 3 \mathrm{H}), 1.08(\mathrm{~d}, J=6.8 \mathrm{~Hz}, 3 \mathrm{H}), 1.00(\mathrm{~d}, J=6.8 \mathrm{~Hz}$, $3 \mathrm{H}), 0.97(\mathrm{~d}, J=6.8 \mathrm{~Hz}, 3 \mathrm{H}), 0.81(\mathrm{~d}, J=6.8 \mathrm{~Hz}, 3 \mathrm{H}), 0.78(\mathrm{~d}, J=6.8 \mathrm{~Hz}, 3 \mathrm{H})$. ${ }^{13} \mathrm{CNMR}\left(125 \mathrm{MHz}, \mathrm{CD}_{3} \mathrm{CN}\right) \delta 164.2,157.2,146.4,133.4,133.2,133.1,132.4,132.2$, $130.1,129.5,120.4,117.6,78.8,78.3,74.9,74.5,62.1,42.8,37.6,36.1,35.2$, 34.9, 33.6, 33.3, 29.6, 22.2, 18.8, 17.1, 16.5, 14.6, 7.9; high resolution mass spectrum $\left(\mathrm{ES}^{+}\right) \mathrm{m} / \mathrm{z}$ $570.3402\left[(\mathrm{M}+\mathrm{Na})^{+}\right.$; calcd for $\left.\mathrm{C}_{31} \mathrm{H}_{49} \mathrm{NO}_{7} \mathrm{Na}: 570.3407\right]$.

\section{Lactone 6}<smiles>C=C/C=C\[C@H](C)[C@H](OC(N)=O)[C@@H](C)[C@@H](O)[C@@H](C)C/C(C)=C\[C@H](C)[C@@H](O)[C@@H](C)/C=C\[C@H](O)C[C@H]1OC(=O)CC[C@H]1C</smiles> 
$[\alpha]_{\mathrm{D}}^{23}+15.4(\mathrm{c} 0.15, \mathrm{MeOH}) ;$ IR 3421, 2963, 1722, 1600, 1382, $1040 \mathrm{~cm}^{-1} ;{ }^{1} \mathrm{HNMR}$ $\left(500 \mathrm{MHz}, \mathrm{CD}_{3} \mathrm{CN}\right) \delta 6.68(\mathrm{ddd}, J=16.9,10.7,10.7 \mathrm{~Hz}, 1 \mathrm{H}), 6.08(\mathrm{dd}, J=11.1,11.1$ $\mathrm{Hz}, 1 \mathrm{H}), 5.56$ (dd, $J=10.5,10.5 \mathrm{~Hz}, 1 \mathrm{H}), 5.42(\mathrm{~m}, 2 \mathrm{H}), 5.25$ (apparent d, $J=16.7 \mathrm{~Hz}$, $1 \mathrm{H}), 5.15$ (d, $J=10.2 \mathrm{~Hz}, 1 \mathrm{H}), 5.05(\mathrm{br} \mathrm{s}, 2 \mathrm{H}), 4.96$ (d, $J=10.0 \mathrm{~Hz}, 1 \mathrm{H}), 4.73$ (dd, $J=$ $8.2,4.0 \mathrm{~Hz}, 1 \mathrm{H}), 4.43(\mathrm{~m}, 1 \mathrm{H}), 4.16$ (ddd, $J=10.2,10.2,1.6 \mathrm{~Hz}, 1 \mathrm{H}), 3.08(\mathrm{~m}, 1 \mathrm{H}), 3.03$ $(\mathrm{m}, 2 \mathrm{H}), 2.73(\mathrm{~d}, J=5.4 \mathrm{~Hz}, 1 \mathrm{H}), 2.68(\mathrm{~d}, J=5.5 \mathrm{~Hz}, 1 \mathrm{H}), 2.62(\mathrm{~d}, J=7.5 \mathrm{~Hz}, 1 \mathrm{H}), 2.53$ $(\mathrm{m}, 1 \mathrm{H}), 2.37(\mathrm{~m}, 1 \mathrm{H}), 2.26(\mathrm{~m}, 1 \mathrm{H}), 1.90-1.72(\mathrm{~m}, 2 \mathrm{H}), 1.78-1.56(\mathrm{~m}, 7 \mathrm{H}), 1.59(\mathrm{~s}, 3 \mathrm{H})$, $1.44(\mathrm{~m}, 1 \mathrm{H}), 1.01(\mathrm{~d}, J=6.9 \mathrm{~Hz}, 3 \mathrm{H}), 1.00(\mathrm{~d}, J=6.8 \mathrm{~Hz}, 3 \mathrm{H}), 0.97(\mathrm{~d}, J=6.8 \mathrm{~Hz}, 3 \mathrm{H})$, $0.88(\mathrm{~d}, J=6.8 \mathrm{~Hz}, 3 \mathrm{H}), 0.80(\mathrm{~d}, J=6.8 \mathrm{~Hz}, 3 \mathrm{H}), 0.74(\mathrm{~d}, J=6.8 \mathrm{~Hz}, 3 \mathrm{H}),{ }^{13} \mathrm{CNMR}(125$ $\left.\mathrm{MHz}, \mathrm{CD}_{3} \mathrm{CN}\right) \delta 171.1,157.3,133.2,132.9,132.8,132.7,132.2,130.1,129.5,117.9$, $81.4,78.8,78.3,74.9,62.2,41.5,37.4,36.1,35.2,35.1,33.6,33.2,32.6,29.1,27.5,22.2$, 18.8, 17.1, 16.7, 16.6, 14.6, 8.0; high resolution mass spectrum $\left(\mathrm{ES}^{+}\right) \mathrm{m} / \mathrm{z} 586.3726$ $\left[(\mathrm{M}+\mathrm{Na})^{+}\right.$; calcd for $\mathrm{C}_{32} \mathrm{H}_{53} \mathrm{NO}_{7} \mathrm{Na}$ : 586.3720].

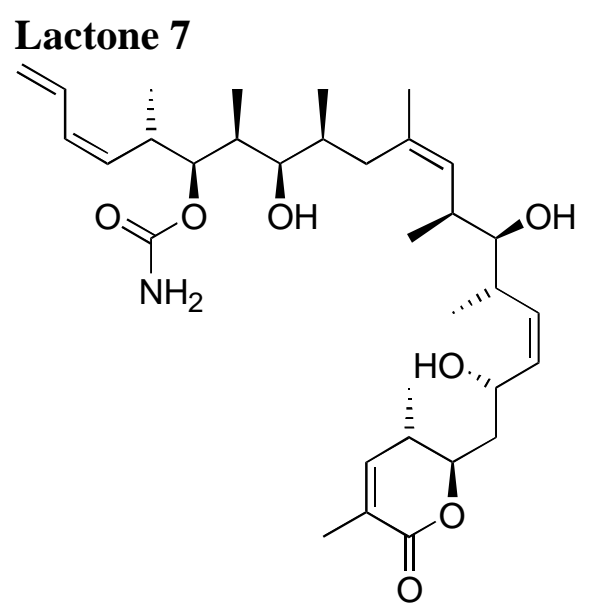

$[\alpha]_{\mathrm{D}}{ }^{23}+77.7^{\circ}(c 0.22 ;) \mathrm{IR}(\mathrm{NaCl}) 3420,29642924,2872,1699,1387,1320,1043,971$ $\mathrm{cm}^{-1} ;{ }^{1} \mathrm{H}$ NMR (500 MHz, $\mathrm{CDCl}_{3}$ ) $\delta 6.68$ (dddd, $J=17.3,6.8,6.8,0.9 \mathrm{~Hz}, 1 \mathrm{H}$ ), 6.43 (dd, $J=2.9,1.4 \mathrm{~Hz}, 1 \mathrm{H}), 6.05(\mathrm{dd}, J=11.1,11.1 \mathrm{~Hz}, 1 \mathrm{H}), 5.47(\mathrm{dd}, J=10.4,10.3 \mathrm{~Hz}, 1 \mathrm{H})$, $5.45(\mathrm{~s}, 1 \mathrm{H}), 5.42(\mathrm{dd}, J=10.7,10.5 \mathrm{~Hz}, 1 \mathrm{H}), 5.35(\mathrm{dd}, J=11.0,8.8 \mathrm{~Hz}, 1 \mathrm{H}), 5.23(\mathrm{dd}, J$ $=16.9,2.0 \mathrm{~Hz}, 1 \mathrm{H}), 5.15-5.04(\mathrm{br} \mathrm{s}, 2 \mathrm{H}), 5.14(\mathrm{~d}, J=10.2 \mathrm{~Hz}, 1 \mathrm{H}), 5.08(\mathrm{~d}, J=10.2 \mathrm{~Hz}$, $1 \mathrm{H}), 4.70(\mathrm{dd}, J=6.8,5.1 \mathrm{~Hz}, 1 \mathrm{H}), 4.53(\mathrm{dd}, J=14.9,6.8 \mathrm{~Hz}, 1 \mathrm{H}), 4.18(\mathrm{ddd}, J=9.1$, $8.0,3.9 \mathrm{~Hz}, 1 \mathrm{H}), 3.17$ (brs, $3 \mathrm{H}) 3.12-3.02(\mathrm{~m}, 2 \mathrm{H}), 2.67-2.62(\mathrm{~m}, 2 \mathrm{H}), 2.54$ (ddddd, $J=$ 7.2, 7.2, 7.2, 5.0, $2.6 \mathrm{~Hz}, 1 \mathrm{H}), 2.47-2.40(\mathrm{~m} .1 \mathrm{H}), 1.94(\mathrm{ddd}, J=5.0,2.5,2.5 \mathrm{~Hz}, 1 \mathrm{H})$, $1.76(\mathrm{~m}, 3 \mathrm{H}), 1.72(\mathrm{dd}, J=12.2,3.6 \mathrm{~Hz}, 1 \mathrm{H}), 1.59(\mathrm{~d}, J=1.2 \mathrm{~Hz}, 3 \mathrm{H}), 1.06(\mathrm{~d}, J=7.2$ $\mathrm{Hz}, 3 \mathrm{H}), 0.98(\mathrm{~d}, J=6.9 \mathrm{~Hz}, 3 \mathrm{H}), 0.95(\mathrm{~d}, J=6.8 \mathrm{~Hz}, 3 \mathrm{H}), 0.87(\mathrm{~d}, J=6.8 \mathrm{~Hz}, 3 \mathrm{H}), 0.86$ 
$(\mathrm{d}, J=6.7 \mathrm{~Hz}, 3 \mathrm{H}), 0.76(\mathrm{~m}, 3 \mathrm{H}), 0.76(\mathrm{~s}, 3 \mathrm{H}) ;{ }^{13} \mathrm{C} \mathrm{NMR}\left(125 \mathrm{MHz}, \mathrm{CDCl}_{3}\right) \delta 165.1$, 157.2 , 154.7, 146.0, 134.5, 133.5, 132.3, 132.2, 131.9, 130.5, 129.3, 117.3, 81.1, 78.5, 77.9, 75.0, 64.0, 40.6, 37.4, 35.8, 35.3, 34.8, 33.9, 33.4, 33.2, 22.4, 17.8, 17.0, 15.9, 15.9, 14.9, 13.8, 8.5; high resolution mass spectrum $\left(\mathrm{ES}^{+}\right) \mathrm{m} / \mathrm{z} 598.3707\left[(\mathrm{M}+\mathrm{Na})^{+}\right.$; calcd for $\mathrm{C}_{33} \mathrm{H}_{53} \mathrm{NNaO}_{7}$ : 598.3720].

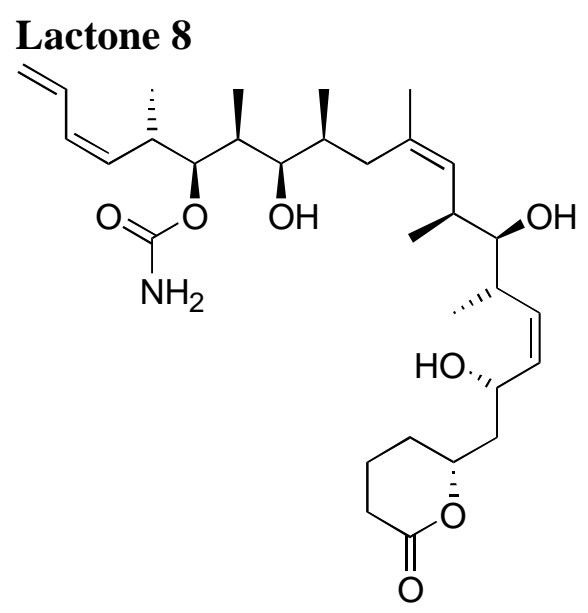

$[\alpha]_{\mathrm{D}}^{23}+22.5(\mathrm{c} 0.08, \mathrm{MeOH}) ;$ IR 3408, 2962, 1711, 1390, $1042 \mathrm{~cm}^{-1} ;{ }^{1} \mathrm{HNMR}(500 \mathrm{MHz}$ $\left.\mathrm{CD}_{3} \mathrm{CN}\right) \delta 6.68(\mathrm{ddd}, J=16.9,10.7,10.7 \mathrm{~Hz}, 1 \mathrm{H}), 6.08(\mathrm{dd}, J=11.2,11.2 \mathrm{~Hz}, 1 \mathrm{H}), 5.56$ $(\mathrm{dd}, J=10.1,10.1 \mathrm{~Hz}, 1 \mathrm{H}), 5.46-5.38(\mathrm{~m}, 2 \mathrm{H}), 5.25(\mathrm{dd}, J=16.7,2.2 \mathrm{~Hz}, 1 \mathrm{H}), 5.15(\mathrm{~d}, J$ $=10.0 \mathrm{~Hz}, 1 \mathrm{H}), 5.07$ (br s, 2H), $4.99(\mathrm{~d}, J=10.0 \mathrm{~Hz}, 1 \mathrm{H}), 4.73(\mathrm{dd}, J=7.8,4.1 \mathrm{~Hz}, 1 \mathrm{H})$, $4.51(\mathrm{~m}, 1 \mathrm{H}), 4.41(\mathrm{~m}, 1 \mathrm{H}), 3.16(\mathrm{ddd}, J=6.7,6.7,4.1 \mathrm{~Hz}, 1 \mathrm{H}), 3.08(\mathrm{~m}, 2 \mathrm{H}), 2.72(\mathrm{~d}, J$ $=5.2 \mathrm{~Hz}, 1 \mathrm{H}), 2.63(\mathrm{~m}, 1 \mathrm{H}), 2.62(\mathrm{~d}, J=5.2 \mathrm{~Hz}, 1 \mathrm{H}), 2.57(\mathrm{~d}, J=6.7 \mathrm{~Hz}, 1 \mathrm{H}), 2.50(\mathrm{~m}$, $1 \mathrm{H}), 2.36(\mathrm{dd}, J=7.8,7.8 \mathrm{~Hz}, 1 \mathrm{H}), 2.30(\mathrm{~m}, 1 \mathrm{H}), 1.90-1.60(\mathrm{~m}, 9 \mathrm{H}), 1.61(\mathrm{~s}, 3 \mathrm{H}), 1.50-$ $1.50(\mathrm{~m}, 1 \mathrm{H}), 1.01(\mathrm{~d}, J=6.7 \mathrm{~Hz}, 3 \mathrm{H}), 0.96(\mathrm{~d}, J=6.8 \mathrm{~Hz}, 3 \mathrm{H}), 0.89$ (d, $J=6.8 \mathrm{~Hz}, 3 \mathrm{H})$, $0.82(\mathrm{~d}, J=6.8 \mathrm{~Hz}, 3 \mathrm{H}), 0.75(\mathrm{~d}, J=6.8 \mathrm{~Hz}, 3 \mathrm{H}) ;{ }^{13} \mathrm{CNMR}\left(125 \mathrm{MHz}, \mathrm{CD}_{3} \mathrm{CN}\right) \delta 171.3$, 157.3 , 133.3, 133.2, 132.9, 132.6, 132.2, 130.1, 129.5, 117.5, 78.8, 78.2, 76.4, 75.0, 62.5, 43.7, 37.5, 36.0, 35.3, 35.1, 33.7, 33.2, 29.0, 28.1, 22.2, 18.6, 18.1, 17.1, 16.3, 14.4, 8.0; high resolution mass spectrum $\left(\mathrm{ES}^{+}\right) \mathrm{m} / \mathrm{z}$ 572.3539. [(M+Na) ${ }^{+}$; calcd for $\mathrm{C}_{31} \mathrm{H}_{51} \mathrm{NO}_{7} \mathrm{Na}$ : 572.3563].

\section{Butyrolactone 9}




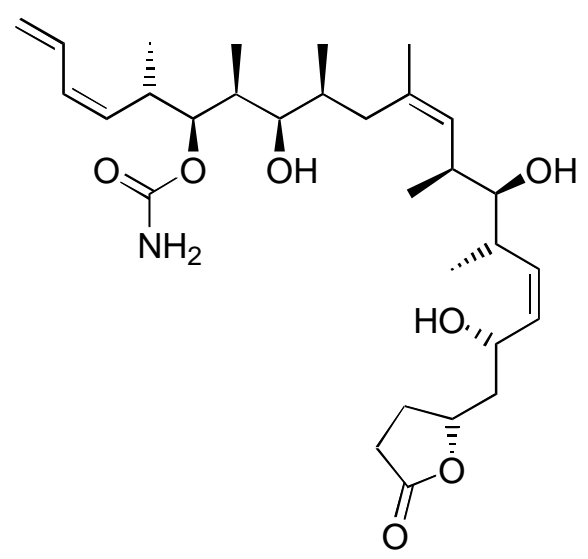

${ }^{1} \mathrm{HNMR}\left(400 \mathrm{MHz}, \mathrm{CDCl}_{3}\right) \delta 6.61(\mathrm{dt}, J=17.0,10.5 \mathrm{~Hz}, 1 \mathrm{H}), 6.03(\mathrm{t}, J=11.0 \mathrm{~Hz}, 1 \mathrm{H})$, 5.53-5.42 (m, 2H), $5.36(\mathrm{t}, J=10.5 \mathrm{~Hz}, 1 \mathrm{H}), 5.21(\mathrm{~d}, J=17.0 \mathrm{~Hz}, 1 \mathrm{H}), 5.14(\mathrm{~m}, 2 \mathrm{H})$, $4.80(\mathrm{~m}, 4 \mathrm{H}), 4.60(\mathrm{~m}, 1 \mathrm{H}), 3.27(\mathrm{t}, J=5.0 \mathrm{~Hz}, 1 \mathrm{H}), 3.19(\mathrm{dd}, J=6.5,5.0 \mathrm{~Hz}, 1 \mathrm{H}), 3.00$ $(\mathrm{dt}, J=10.0,7.0 \mathrm{~Hz}, 1 \mathrm{H}), 2.78(\mathrm{dt}, J=9.0,7.0 \mathrm{~Hz}, 1 \mathrm{H}), 2.59-2.52(\mathrm{~m}, 2 \mathrm{H}), 2.37(\mathrm{~m}$, $1 \mathrm{H}), 2.03(\mathrm{~m}, 2 \mathrm{H}), 1.95-1.77(\mathrm{~m}, 6 \mathrm{H}), 1.64(\mathrm{~d}, J=1.0 \mathrm{~Hz}, 3 \mathrm{H}), 1.01(\mathrm{~d}, J=7.0 \mathrm{~Hz}, 3 \mathrm{H})$, 1.01-0.97 (m, 6H), $0.94(\mathrm{~d}, J=7.0 \mathrm{~Hz}, 3 \mathrm{H}), 0.82(\mathrm{~d}, J=5.5 \mathrm{~Hz}, 3 \mathrm{H}) ;{ }^{13} \mathrm{CNMR}(100$ $\left.\mathrm{MHz}, \mathrm{CDCl}_{3}\right) \delta 177.2,157.3,134.6,133.5,133.5,133.4,132.5,129.8,129.6,117.9$, 78.9, 78.7, 78.0, 75.8, 65.2, 43.2, 37.2, 35.8, 35.8, 35.2, 34.6, 33.0, 28.8, 28.4, 23.2, 18.4, $17.4,15.6,13.8,8.8 ; \mathrm{m} / \mathrm{z} 558[\mathrm{M}+\mathrm{Na}]^{+}, 519$; high resolution mass spectrum $\left(\mathrm{ES}^{+}\right) \mathrm{m} / \mathrm{z}$ $558.3425\left[(\mathrm{M}+\mathrm{Na})^{+}\right.$; calcd for $\mathrm{C}_{30} \mathrm{H}_{49} \mathrm{NNaO}_{7}$ : 558.3401].

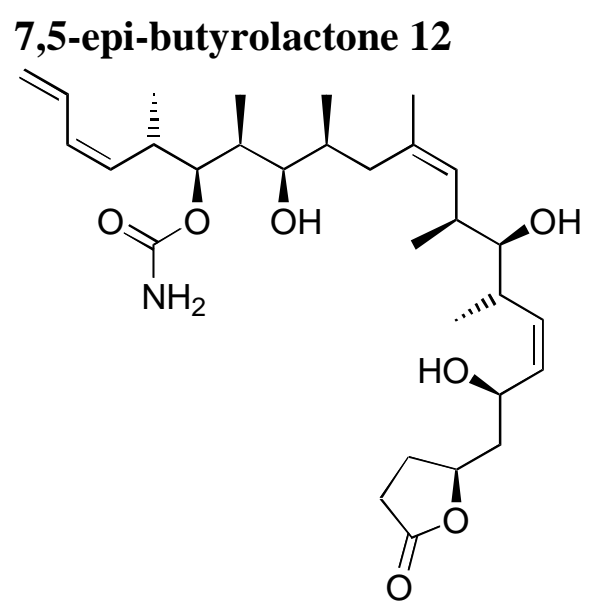

${ }^{1} \mathrm{HNMR}\left(400 \mathrm{MHz}, \mathrm{CDCl}_{3}\right) \delta 6.61(\mathrm{dt}, J=17.0,10.5 \mathrm{~Hz}, 1 \mathrm{H}), 6.02(\mathrm{t}, J=11.0 \mathrm{~Hz}, 1 \mathrm{H})$, $5.51(\mathrm{dd}, J=11.0,8.5 \mathrm{~Hz}, 1 \mathrm{H}), 5.41(\mathrm{~m}, 2 \mathrm{H}), 5.20(1 \mathrm{H}, \mathrm{d}, J=17.0 \mathrm{~Hz}, \mathrm{H}-24$ trans $), 5.15$ $(\mathrm{d}, J=9.5 \mathrm{~Hz}, 1 \mathrm{H}), 5.11(\mathrm{~d}, J=10.5 \mathrm{~Hz}, 1 \mathrm{H}), 4.81(\mathrm{~m}, 1 \mathrm{H}), 4.70(\mathrm{br} \mathrm{s}, 2 \mathrm{H}), 4.68(\mathrm{dd}, J=$ 8.0, $3.0 \mathrm{~Hz}, 1 \mathrm{H}), 4.56(\mathrm{td}, J=9.0,3.0 \mathrm{~Hz}, 1 \mathrm{H}), 3.33(\mathrm{dd}, J=6.5,3.5 \mathrm{~Hz}, 1 \mathrm{H}), 3.17(\mathrm{dd}, J$ 
$=8.0,4.0 \mathrm{~Hz}, 1 \mathrm{H}), 2.98(\mathrm{~m}, 1 \mathrm{H}), 2.71-2.65(\mathrm{~m}, 2 \mathrm{H}), 2.55(\mathrm{dd}, J=10.0,9.5 \mathrm{~Hz}, 1 \mathrm{H}), 2.36$ $(\mathrm{td}, J=13.0,6.5 \mathrm{~Hz}, 1 \mathrm{H}), 2.04-1.93(\mathrm{~m}, 2 \mathrm{H}), 1.92-1.86(\mathrm{~m}, 4 \mathrm{H}), 1.83-1.72(\mathrm{~m}, 2 \mathrm{H}), 1.68$ (s, 3H), $1.01(\mathrm{~d}, J=7.0 \mathrm{~Hz}, 3 \mathrm{H}), 0.99-0.97(\mathrm{~m}, 9 \mathrm{H}), 0.83(\mathrm{~d}, J=6.0 \mathrm{~Hz}, 3 \mathrm{H}),{ }^{13} \mathrm{CNMR}$ $\left(100 \mathrm{MHz}, \mathrm{CDCl}_{3}\right) \delta 177.3,157.0,135.7,134.6,133.9,132.9,132.1,129.9,129.0,117.8$, 78.3, 78.3, 77.8, 75.4, 63.1, 43.0, 37.2, 36.2, 35.6, 35.1, 34.7, 32.8, 28.9, 28.4, 23.3, 17.6, $17.3,15.6,13.2,9.0 ; \mathrm{m} / \mathrm{z} 558[\mathrm{M}+\mathrm{Na}]^{+}, 518,457$; high resolution mass spectrum $\left(\mathrm{ES}^{+}\right)$ $\mathrm{m} / \mathrm{z} 558.3415\left[(\mathrm{M}+\mathrm{Na})^{+}\right.$; calcd for $\left.\mathrm{C}_{30} \mathrm{H}_{49} \mathrm{NNaO}_{7}: 558.3401\right]$.

\section{Benzobutyrolactone 13}

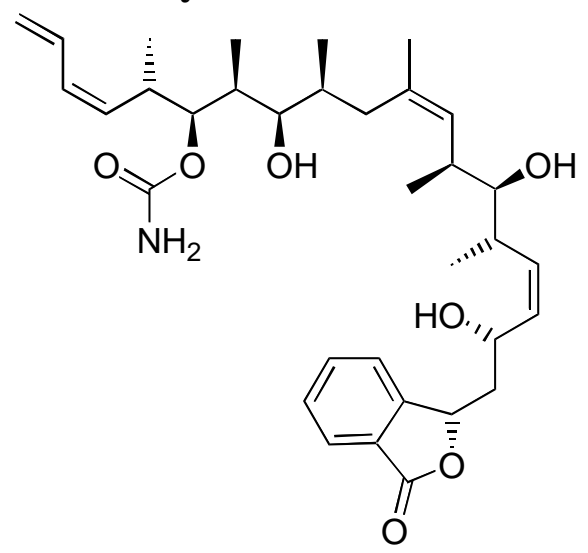

${ }^{1} \mathrm{HNMR}\left(400 \mathrm{MHz}, \mathrm{CDCl}_{3}\right) \delta 7.90(\mathrm{~d}, J=7.5 \mathrm{~Hz}, 1 \mathrm{H}), 7.68(\mathrm{dd}, J=7.5,7.0 \mathrm{~Hz}, 1 \mathrm{H})$, $7.53(\mathrm{dd}, J=7.5,7.0 \mathrm{~Hz}, 1 \mathrm{H}), 7.46(\mathrm{~d}, J=7.5 \mathrm{~Hz}, 1 \mathrm{H}), 6.61(\mathrm{dt}, J=17.0,10.5 \mathrm{~Hz}, 1 \mathrm{H})$, $6.02($ app t $, J=11.0 \mathrm{~Hz}, 1 \mathrm{H}), 5.78(\mathrm{~d}, J=9.0 \mathrm{~Hz}, 1 \mathrm{H}), 5.57-5.48(\mathrm{~m}, 2 \mathrm{H}), 5.34(\operatorname{app~t}, J=$ $10.5 \mathrm{~Hz}, 1 \mathrm{H}), 5.22(\mathrm{~d}, J=17.0 \mathrm{~Hz}, 1 \mathrm{H}), 5.13(\mathrm{~m}, 2 \mathrm{H}), 4.82(\mathrm{t}, J=7.0 \mathrm{~Hz}, 1 \mathrm{H}), 4.69(\mathrm{~m}$, $3 \mathrm{H}), 3.21(\mathrm{~m}, 2 \mathrm{H}), 2.96(\mathrm{dt}, J=10.5,7.0 \mathrm{~Hz}, 1 \mathrm{H}), 2.82(\mathrm{~m}, 1 \mathrm{H}), 2.55(\mathrm{~m}, 1 \mathrm{H}), 2.21$ (ddd, $J=14.5,10.5,2.5 \mathrm{~Hz}, 1 \mathrm{H}), 2.00(\mathrm{~m}, 1 \mathrm{H}), 1.82(\mathrm{~m}, 2 \mathrm{H}), 1.72(\mathrm{ddd}, J=14.0,11.0,3.0 \mathrm{~Hz}$, $1 \mathrm{H}), 1.67(\mathrm{~m}, 1 \mathrm{H}), 1.56(\mathrm{~s}, 3 \mathrm{H}), 1.04(\mathrm{~d}, J=6.5 \mathrm{~Hz}, 3 \mathrm{H}), 0.97(\mathrm{~d}, J=7.0 \mathrm{~Hz}, 3 \mathrm{H}), 0.94$ $(\mathrm{d}, J=7.0 \mathrm{~Hz}, 3 \mathrm{H}), 0.85(\mathrm{~d}, J=7.0 \mathrm{~Hz}, 3 \mathrm{H}), 0.80(\mathrm{~d}, J=5.0 \mathrm{~Hz}, 3 \mathrm{H}) ;{ }^{13} \mathrm{CNMR}(100$ $\left.\mathrm{MHz}, \mathrm{CDCl}_{3}\right) \delta 170.4,157.1,150.1,134.7,134.1,133.6,133.6,132.4,132.0,131.8$, $129.8,129.4,129.1,125.8,121.7,117.9,78.9,78.7,78.3,75.8,65.3,42.8,37.2,35.8$, $35.8,35.3,34.6,33.0,23.1,18.4,17.4,15.8,13.8,8.6 ; \mathrm{m} / z 606[\mathrm{M}+\mathrm{Na}]^{+}, 567[\mathrm{M}+\mathrm{H}]^{+}$, 506; high resolution mass spectrum $\left(\mathrm{ES}^{+}\right) \mathrm{m} / \mathrm{z} 606.3423\left[(\mathrm{M}+\mathrm{Na})^{+}\right.$; calcd for $\mathrm{C}_{34} \mathrm{H}_{49} \mathrm{NNaO}_{7}$ : 606.3401]. 